\title{
Cold H I in turbulent eddies and galactic spiral shocks
}

\author{
Steven J. Gibson ${ }^{1}$, A. Russell Taylor ${ }^{2}$, Jeroen M. Stil ${ }^{2}$, Christopher \\ M. Brunt ${ }^{3}$, Dain W. Kavars ${ }^{4}$ and John M. Dickey ${ }^{4,5}$ \\ ${ }^{1}$ Arecibo Observatory, National Astronomy and Ionosphere Center, Arecibo, PR 00612, U.S.A. \\ ${ }^{2}$ Dept. of Physics \& Astronomy, University of Calgary, Calgary, Alberta T2N 1N4, Canada \\ ${ }^{3}$ School of Physics, University of Exeter, Exeter, United Kingdom EX4 4QL \\ ${ }^{4}$ Department of Astronomy, University of Minnesota, Minneapolis, MN 55455, U.S.A. \\ ${ }^{5}$ School of Mathematics and Physics, University of Tasmania, Hobart, TAS 7001, Australia
}

\begin{abstract}
H I 21cm-line self-absorption (HISA) reveals the shape and distribution of cold atomic clouds in the Galactic disk. Many of these clouds lack corresponding $\mathrm{CO}$ emission, despite being colder than purely atomic gas in equilibrium models. HISA requires background line emission at the same velocity, hence mechanisms that can produce such backgrounds. Weak, small-scale, and widespread absorption is likely to arise from turbulent eddies, while strong, large-scale absorption appears organized in cloud complexes along spiral arm shocks. In the latter, the gas may be evolving from an atomic to a molecular state prior to star formation, which would account for the incomplete HISA-CO agreement.
\end{abstract}

Keywords. radiative transfer, surveys, ISM: clouds, ISM: evolution, ISM: kinematics and dynamics, ISM: structure, Galaxy: structure, radio lines: ISM

\section{Imaging the cold atomic medium}

Cold atomic gas contains a large fraction of the mass of the interstellar medium (ISM) and is a critical precursor to molecular cloud formation. However, this "cold atomic medium" is hard to map in isolation, since warmer gas is often brighter in traditional H I 21cm-line emission observations. Fortunately, with proper angular resolution, cold atomic clouds can be imaged as H I self-absorption (HISA) against warmer background H I emission (Gibson et al. 2000). Recent large-scale radio synthesis surveys like the Canadian and VLA Galactic plane surveys (CGPS: Taylor et al. 2003; VGPS: Stil et al. 2006) are both well suited to HISA studies.

Figure 1 compares some sample CGPS HISA to CO emission. The intricate structure of the HISA clouds is clear, as is their frequent lack of apparent CO. This lack is a puzzle unless significant $\mathrm{H}_{2}$ is present without $\mathrm{CO}$, since the low temperatures of many HISA features $(T<50 \mathrm{~K})$ are hard to explain without molecular gas. But if these clouds are evolving rather than stable objects, then perhaps many have not yet formed enough $\mathrm{CO}$ to detect (e.g., Klaassen et al. 2005).

Using an algorithm to identify and extract HISA features in the $\mathrm{H}$ I data, we recently published a HISA census of the $73^{\circ} \times 9^{\circ}$ area covered by the first 5 years of the CGPS (Gibson et al. 2005). As shown in Figure 2, a low-level froth of weak, disorganized HISA is found throughout the regions of the CGPS where the background emission is bright enough for the HISA to be reliably detected. By contrast, stronger absorption is organized into discrete clouds and complexes. 

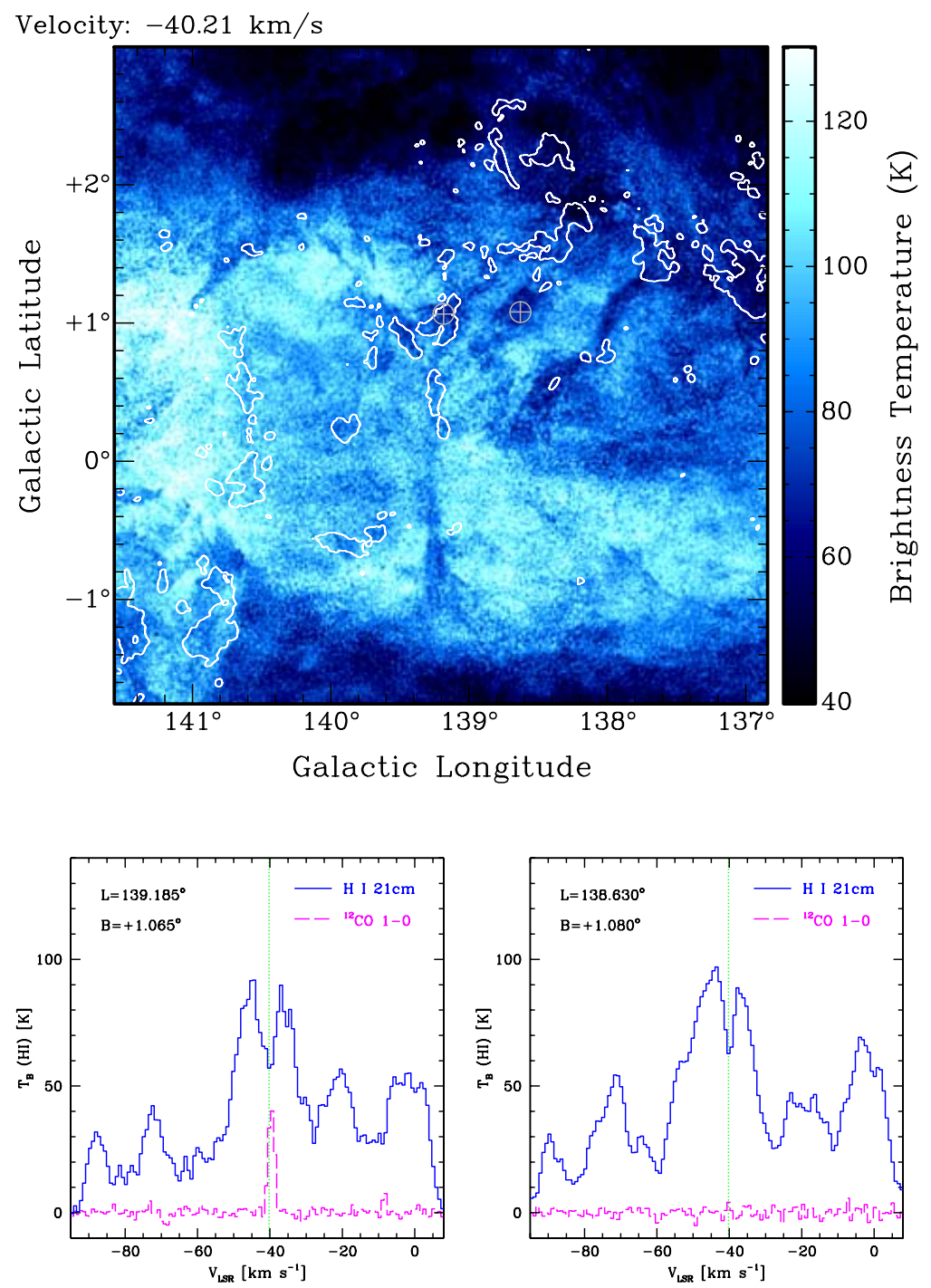

Figure 1. Sample HISA in the CGPS. The upper panel shows an $\mathrm{H}$ I channel map with ${ }^{12} \mathrm{CO}$ 1-0 contours from Heyer et al. (1998); the LSR velocity places this gas in the Perseus spiral arm some $2 \mathrm{kpc}$ away. $\mathrm{H}$ I and $\mathrm{CO}$ spectra at the two marked positions are plotted in the lower panels, showing that HISA is found with and without CO, with the latter case more common in the outer Galaxy.

\section{Velocity perturbation mechanisms}

HISA requires background line emission at the same radial velocity as the foreground cloud. Consequently, HISA radiative transfer probes both the temperature and the velocity field of Galactic H I. The CGPS is primarily in the outer Galaxy, where pure differential rotation allows only one position along the line of sight to have a particular velocity. Since we detect HISA in the outer Galaxy, the real velocity field must be 

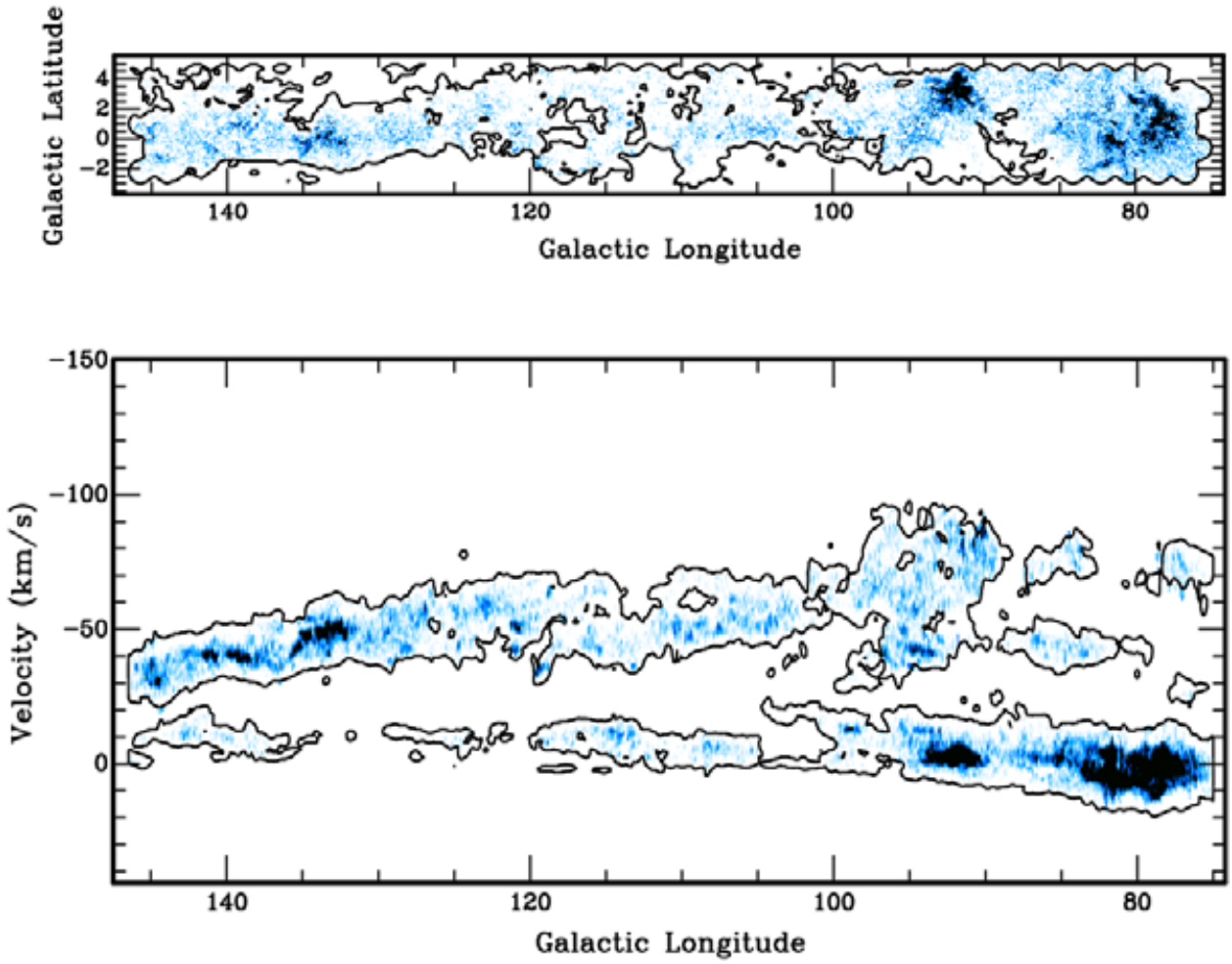

Figure 2. Longitude-latitude and longitude-velocity maps of HISA extracted from the initial $73^{\circ} \times 9^{\circ}$ portion of the CGPS, in which the HISA ON-OFF absorption amplitude is integrated over velocity (top) and latitude (bottom), with stronger absorption being darker. Contours mark where the $\mathrm{H}$ I emission background becomes too faint for reliable HISA detection.

perturbed from this simple rotation model to provide background fields to absorb against. Two known mechanisms for this are turbulence and spiral density waves.

The weak, widespread HISA in Figure 2 can be explained as an ambient froth of cold atomic gas in the ISM made visible by turbulent eddies; perhaps the cold gas is even a product of convergent turbulent flows, as some models suggest (e.g., Vázquez-Semadeni et al. 2006). The stronger HISA, however, is concentrated into distinct complexes, especially along the Perseus arm near $-40 \mathrm{~km} \mathrm{~s}^{-1}$, arguing for a more organized mechanism for these features. The very cold temperatures implied by this strong absorption could arise from gas that is forming $\mathrm{H}_{2}$ but has not yet formed much $\mathrm{CO}$.

\section{Interpreting the galactic HISA distribution}

Figure 3 shows a new HISA survey incorporating the VGPS and extensions to the CGPS (Gibson et al. 2006). This plot includes CO contours and a curve marking the maximum velocity departure from circular rotation predicted by Roberts (1972) for the Perseus spiral shock. Apart from some irregular ISM structure Roberts did not model, the strong Perseus HISA lies near the shock curve but at less extreme velocities, consistent with clouds lying just downstream of the spiral shock. Similar but fainter shock-related HISA may be seen in the Outer arm at negative velocities in the VGPS data. Both arms have a poor HISA-CO match, as would be expected for evolving gas; in this scenario, 


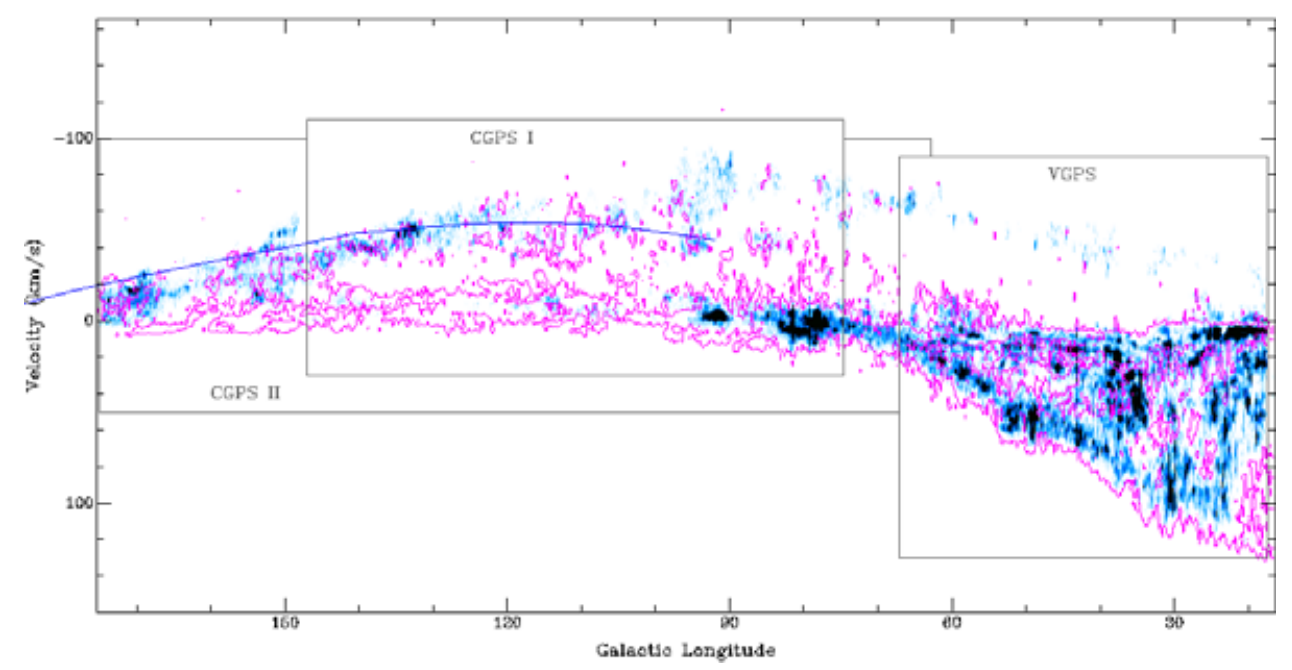

Figure 3. Longitude-velocity HISA distribution over the entire area covered by the original CGPS, the CGPS II extension, and the VGPS. Contours show molecular gas traced by Dame et al. (2001) ${ }^{12} \mathrm{CO} 1-0$ emission, and the curved line marks the maximum velocity departure from Galactic rotation predicted by the Perseus arm spiral shock model of Roberts (1972).

the visible $\mathrm{CO}$ traces more evolved gas further downstream, where little background $\mathrm{H}$ I emission is left to show the remaining cold atomic gas in the CO clouds as HISA.

In the inner Galaxy, the picture is more complex. A much larger amount of HISA is seen, since even simple rotation provides near and far points on each sight line with the same velocity. This allows a much more widespread H I emission background, which may account for the stronger HISA-CO agreement and the lack of clearly-defined spiral arms. However, the latter requires a significant amount of cold interarm $\mathrm{H}$ I in the disk. Whether the interarm cold cloud population is related to the outer-Galaxy turbulent HISA population is still under investigation.

\section{Acknowledgements}

This work has been supported by the National Astronomy and Ionosphere Center operated by Cornell University under Cooperative Agreement with the U.S. National Science Foundation and by grants from the Natural Sciences and Engineering Research Council of Canada to the University of Calgary.

\section{References}

Dame, T. M., Hartmann, D. \& Thaddeus, P. 2001, ApJ 547, 792

Gibson, S. J., Taylor, A. R., Dewdney, P. E. \& Higgs, L. A. 2000, ApJ 540, 851

Gibson, S. J., Taylor, A. R., Stil, J. M., Brunt, C. M., Kavars, D. W. \& Dickey, J. M. 2006, in preparation

Gibson, S. J., Taylor, A. R., Higgs, L. A., Brunt, C. M. \& Dewdney, P. E. 2005, ApJ 626, 195

Heyer, M. H., Brunt, C., Snell, R. L., Howe, J. E., Schloerb, F. P. \& Carpenter, J. M. 1998, ApJS 115, 241

Klaassen, P. D., Plume, R., Gibson, S. J., Taylor, A. R. \& Brunt, C. M. 2005, ApJ 631, 1001

Roberts, W. W. 1972, ApJ 173, 259

Stil, J. M., Taylor, A. R., Dickey, J. M., Kavars, D. A., Martin, P. G., Rothwell, T. A., Boothroyd, A., Lockman, F. J. \& McClure-Griffiths, N. M. 2006, AJ 132, 1158

Taylor, A. R., et al. 2003, AJ 125, 3145

Vázquez-Semadeni, E., Ryu, D., Passot, T., González, R. F. \& Gazol, A. 2006, ApJ 643, 245 


\section{Discussion}

FUKUI: An important aspect of cold HI is that we can detect enveloping lower density gas that is not detectable in CO. This part of the mostly atomic gas will be important to study cloud formation, and sub-mm CI surveys on a large scale will be coming soon to provide more knowledge of this part of the less dense gas.

GiBson: Thank you. I'm pleased to hear of the coming CI surveys, which should be very useful for probing the state of the gas. I will only add that the cold HI does not always appear in CO envelopes; it can also be seen in cores or even throughout the cloud, depending on the object. In many cases of course, it appears without $\mathrm{CO}$ at all, in which case the molecular content of the cloud is unclear.

ELMEGREEN: I think the lack of CO in the outer galaxy HISA is not surprising considering the general gradient of molecular fraction with radius. This is presumably the result of a pressure gradient because low pressure regions have low density cloud envelopes and then a high column density is needed to shield molecules against photodissociation.

GiBson: That is a very good point. However, are you saying that we should expect no $\mathrm{H} 2$, or merely no CO? If there are no molecules at all, then it becomes more difficult to explain some of the very low temperatures found for HISA clouds, which are typically less than $50 \mathrm{~K}$ and can be much less in some cases. To me the most likely explanation in this case is that the clouds are unstable, transitional objects. 